\title{
Área Foliar de DuAs Trepadeiras Infestantes de CANA-DE-Açúcar UTILIZANdo Dimensóes Lineares De FolHas ${ }^{1}$
}

\author{
Foliar Area Estimate of Two Sugarcane-infesting Weeds using Leaf Blade Linear Dimensions
}

CARDOZO, N.P. ${ }^{2}$, PARREIRA, M.C. ${ }^{3}$, ALVES, P.L.C.A. ${ }^{4}$ e BIANCO, S. ${ }^{4}$

\begin{abstract}
RESUMO - Esta pesquisa teve como objetivo obter uma equação, por meio de medidas lineares dimensionais das folhas, que permitisse a estimativa da área foliar de Momordica charantia e Pyrostegia venusta. Entre maio e dezembro de 2007, foram estudadas as correlações entre a área folia real (Sf) e as medidas dimensionais do limbo foliar, como o comprimento ao longo da nervura principal (C) e a largura máxima (L) perpendicular à nervura principal. Todas as equações, exponenciais geométricas ou lineares simples, permitiram boas estimativas da área foliar. Do ponto de vista prático, sugere-se optar pela equação linear simples envolvendo o produto $\mathrm{C}$ x L, considerando-se o coeficiente linear igual a zero. Desse modo, a estimativa da área foliar de Momordica charantia pode ser feita pela fórmula Sf = 0,4963 x (C x L), e a de Pyrostegia venusta, por $\mathrm{Sf}=0,6649 \times(\mathrm{C} \times \mathrm{L})$.
\end{abstract}

Palavras-chave: plantas daninhas, análise de crescimento, estimativa.

ABSTRACT - The aim of this study was to obtain a mathematical equation to estimate the leaf area of Momordica charantia and Pyrostegia venusta using linearleaf blade measurements. Correlation studies were conducted involving real leaf area (Sf) and leaflength (C), maximum leaf width (L) and $C \times L$. The linear and geometric equations involving parameter C provided good leaf area estimates. From a practical viewpoint, the simple linear equation of the regression model is suggested using the C $x$ L parameter, i.e., considering the linear coefficient equal to zero. Thus, leaf area estimate of Momordica charantia can be obtained by using the equation $S f=0.4963 \times(C \times L)$, and that of Pyrostegia venusta by using equation $S f=0.6649 \times(C \times L)$.

Keywords: weeds, growth analysis, estimative

\section{INTRODUÇÃO}

Com o aumento da mecanização da cultura da cana-de-açúcar, a colheita de forma crua tem promovido o aparecimento de alguns problemas no controle de certas espécies daninhas. Nesse sentido, as plantas daninhas popularmente conhecidas como melão-desão-caetano e cipó-de-são-joão têm representado problema considerável na região de Jaboticabal-SP, dificultando o desenvolvimento da cana-de-açúcar e de sua colheita. Contudo, poucas são as informações existentes sobre essas plantas, as quais carecem de maiores descrições a respeito de sua biologia, morfologia e formas de controle químico.

O melão-de-são-caetano (Momordica charantia), da família Cucurbitaceae, é uma planta anual trepadeira, reproduzida por sementes. Originário da Ásia, é uma planta monoica, com flores amarelas frequentemente pareadas (Kissman et al., 1999). No Brasil, o tipo selvagem é uma planta daninha bastante

Recebido para publicação em 22.10.2008 e na forma revisada em 13.11.2009.

2 Engo-Agr ${ }^{\circ}$., Dep. de Biologia Aplicada à Agronomia, Laboratório de Biologia e Manejo de Plantas Daninhas - FCAV/UNESP; ${ }^{3}$ Engô-Agro ${ }^{-}$, Mestranda em Produção Vegetal, Laboratório de Biologia e Manejo de Plantas Daninhas - FCAV-UNESP, Via de Acesso Prof. Paulo Donato Castelhane, s/n-14884-900 - Jaboticabal-SP; <mariana.parreira@posgrad.fcav.unesp.br>, ${ }^{4}$ Prof. Assistente do Dep. de Biologia Aplicada à Agronomia, Laboratório de Biologia e Manejo de Plantas Daninhas -FCAV-UNESP, <plalves@fcav.unesp.br e silvano.bianco@fcav.unesp.br>. 
frequente em pomares, hortas, cafezais, cercas, alambrados e terrenos baldios (Lorenzi, 2008). Já a espécie Pyrostegia venusta (sinonimia Pyrostegia ignea e Bignonia venusta), conhecida popularmente como cipó ou florde-são-joão, é uma liana trepadeira com expressiva dispersão em quase todo o sul do Brasil, sendo encontrada nas orlas das matas, nos campos, no litoral e na beira das estradas (Lorenzi, 2008). O caule é utilizado como tônico antidiarreico e na confecção de cestos. Pode ser usada como planta ornamental que se multiplica rapidamente, servindo para revestir muros e caramanchões. O cipó-de-são-joão tem uso medicinal, pois possui elevada quantidade de alantoína isolada nas raízes, permitindo o uso dessa espécie como fonte natural para comercialização dessa substância, a qual, principalmente, pode ser utilizada para tratamento de vitiligo (Ferreira et al., 2000).

O controle efetivo de espécies daninhas só é possível com o conhecimento da biologia das espécies envolvidas. Todavia, para muitas espécies ainda não há informações suficientes (Bianco et al., 1995). Diante disso, estudos que elucidem os padrões de crescimento e desenvolvimento de plantas daninhas tornam possivel a compreensão mais adequada de sua ação sobre o ambiente e sua interferência em outras plantas (Lucchesi, 1984).

Desses parâmetros de crescimento vegetal, a área foliar é um dos mais importantes e um dos mais difíceis de ser mensurados (Carvalho \& Christoffoleti, 2007). Nesse sentido, a busca de métodos fáceis de serem executados, rápidos e não destrutivos, que estimem a área foliar com precisão, torna-se importante para avaliar o crescimento das plantas em campo. Esses métodos trazem como vantagem o fato de que as amostragens poderão ser executadas com as mesmas plantas durante o seu ciclo de desenvolvimento, reduzindo o erro experimental associado a procedimentos de amostragens destrutivas (Silva et al., 1998).

As equações matemáticas desenvolvidas para determinação da área foliar dos folíolos são obtidas por modelos de regressão, baseados em medidas lineares. Davis (1940) observou que os foliolos laterais de folhas de feijoeiro apresentaram formato irregular quando comparados com o folíolo central; logo, espera-se maior correlação entre a área do folíolo central e as medidas de largura e comprimento dos folíolos centrais em relação aos folíolos laterais. Benincasa et al. (1976) determinaram fatores de correção, os quais correlacionaram o produto comprimentolargura do folíolo de feijoeiro com a área foliar obtida com planimetro em diferentes intensidades de radiação solar e microclimas.

O objetivo do presente trabalho foi determinar equações que, por meio de dimensões lineares das folhas de Momordica charantia e Pyrostegia venusta, permitam a estimativa da área foliar dessas espécies.

\section{MATERIAL E MÉTODOS}

O experimento foi desenvolvido em condições de laboratório, de maio a dezembro de 2007, no Laboratório de Biologia e Manejo de Plantas Daninhas da FCAV-UNESP, no Departamento de Biologia Aplicada à Agropecuária, com as seguintes coordenadas geográficas: latitude de $22^{\circ} 42^{\prime} \mathrm{S}$, longitude de $47^{\circ} 37^{\prime} \mathrm{W}$ e altitude de 546 metros.

Foram coletadas 200 folhas de Momordica charantia e 200 de Pyrostegia venusta, de plantas sujeitas às mais diversas condições de clima da região. Empregaram-se todas as folhas que se apresentavam integras, sem deformações oriundas de fatores climáticos, de pragas ou doenças.

O método baseou-se nas dimensões comprimento $(\mathrm{C})$ e largura $(\mathrm{L})$ das folhas. O comprimento foi definido como a distância entre o ponto de inserção do pecíolo no limbo foliar e a extremidade oposta da folha, e a largura, como a maior dimensão perpendicular ao eixo do comprimento. As folhas foram coletadas no campo e levadas no mesmo dia ao laboratório, onde a área real, o comprimento e a largura de seus folíolos foram medidos, utilizando-se o medidor "Area Meter" (Licor Inc., Lincon, Nebrasca, US), modelo LICOR LI-3000.

A partir das dimensões foliares foram realizadas regressões com o intuito de obter a equação mais adequada para representar a área foliar das espécies estudadas, empregando as seguintes equações: linear, $\mathrm{Y}=\mathrm{a}+\mathrm{bx}$; 
linear pela origem, $\mathrm{Y}=\mathrm{bx}$; geométrica, $\mathrm{Y}=\mathrm{ax}^{\mathrm{b}}$; e exponencial, $\mathrm{Y}=\mathrm{ab}^{\mathrm{x}} \mathrm{-} \mathrm{o}$ valor $\mathrm{Y}$ estima a área do limbo foliar em função de $\mathrm{X}$, cujos valores podem ser o comprimento $(C)$, a largura (L) ou o produto $\left(C^{*} \mathrm{~L}\right)$. No caso de $\mathrm{X}$ igual a $\left(\mathrm{C}^{*} \mathrm{~L}\right)$, estimou-se também a equação linear passando pela origem, o que significa supor que a área é proporcional a um retângulo $\left(\mathrm{C}^{*} \mathrm{~L}\right)$.

Todas as equações foram lineares ou linearizáveis por transformação, de modo que os ajustes foram feitos a partir de retas. Para realizar as comparações entre os modelos, foram obtidas as somas de quadrados das diferenças entre os valores observados e os preditos pelos modelos, denominando isso de soma de quadrados do resíduo. No caso dos modelos com transformação (geométrica e exponencial), foi feita a volta para escala original e, depois disso, obtidas as referidas somas de quadrados do resíduo.

Os coeficientes de correlação foram os obtidos com as variáveis de trabalho $\mathrm{X} \mathrm{e} \mathrm{Y}$, no caso linear; logaritmo de $\mathrm{Y}$ e logaritmo de $\mathrm{X}$, no caso geométrico; e logaritmo de $\mathrm{Y}$ e $\mathrm{X}$, no caso exponencial. O número de graus de liberdade foi estimado pelo número de folhas analisadas menos o número de parâmetros estimados para cada modelo. Para testar o acréscimo de soma de quadrados do resíduo do modelo passando pela origem, em relação ao modelo com intercepto, utilizou-se o teste $\mathrm{F}$ condicional: $\mathrm{F}=(\mathrm{SQRes} .(0,0)-\mathrm{SQRes} . \mathrm{CL}) /$ SQRes. CL/GL), com 1 e 2 GL (graus de liberdade), em que GL é o número de folhas menos dois (Neter \& Wasserman, 1974; Mead \& Curnow, 1983), sendo SQRes $(0,0)$ igual à soma de quadrados do resíduo do modelo linear passando pela origem (modelo Y $=\mathrm{bx}$ ) e SQRes (CL) igual à soma de quadrados do resíduo do modelo linear com parâmetros a e b $(\mathrm{Y}=\mathrm{a}+\mathrm{bx})$. Como critério para escolha da melhor equação de regressão para estimar a área foliar, foi adotada aquela com menor soma de quadrados do resíduo na escala real (sem transformação) e o maior coeficiente de determinação estimado (Peressin et al., 1984).

\section{RESULTADOS E DISCUSSÃO}

Os resultados das análises de regressão realizados a partir dos valores de comprimento (C), largura (L), área foliar real (Sf) e o produto do comprimento pela largura da folha (C x L) de Momordica charantia e Pyrostegia venusta são apresentados na Tabela 1. Para ambas as espécies, todas as equações obtidas permitiram estimar satisfatoriamente sua área foliar, uma vez que em todos os casos os coeficientes de determinação foram superiores a 0,87 . O menor coeficiente de determinação foi de 0,8754 para Momordica charantia e 0,8912 para Pyrostegia venusta. Esses resultados indicam que 87,54 e $89,12 \%$ das variações observadas nas áreas foliares de Momordica charantia e Pyrostegia venusta, respectivamente, foram explicadas pela equação linear simples, utilizando o comprimento do limbo foliar ao longo da nervura principal.

As regressões lineares simples entre a área foliar real e o produto do comprimento pela largura da folha obtiveram, para as duas espécies, os maiores valores do coeficiente de determinação e os menores valores da soma de quadrados do resíduo - fato que indica serem essas as equações que permitem obter estimativas mais precisas da área foliar das espécies em estudo.

Não houve diferenças significativas entre as equações que representam o produto entre o comprimento e a largura, passando ou não pela origem. Essas equações apresentaram estimativas do coeficiente de determinação da ordem de 0,9460 e 0,9755, respectivamente para Momordica charantia e Pyrostegia venusta, sugerindo, assim, que 94,60 e 97,55\% das variações totais observadas podem ser explicadas pela regressão linear. Como não há alteração expressiva na soma de quadrados do resíduo, a equação linear simples com a reta passando pela origem é a mais recomendada, pois é de mais fácil utilização do ponto de vista prático - fato ressaltado por Bianco et al. (2007).

Assim, a estimativa da área foliar de Momordica charantia pode ser obtida pela equação $\mathrm{Sf}=0,4963 \times(\mathrm{C} \times \mathrm{L})$, ou seja, 49,63\% do produto entre o comprimento e a largura máxima do limbo foliar (Figura 1).

Para Pyrostegia venusta essa estimativa pode ser obtida pela equação $\mathrm{Sf}=0,6649 \mathrm{x}$ (C x L), ou seja, 66,49\% do produto entre o comprimento e a largura máxima do limbo foliar. Houve pequenas dispersões dos dados em 
Tabela 1 - Equações de regressão estimadas, coeficientes de determinação, graus de liberdade e somas de quadrados de desvios da regressão da área foliar, em função das medidas lineares do limbo foliar de Momordica charantia e Pyrostegia venusta. FCAV/UNESP, Jaboticabal-SP, 2007

\begin{tabular}{|c|c|c|c|c|c|}
\hline \multirow{2}{*}{$\mathrm{X}^{1 /}$} & \multirow{2}{*}{ Tipo de equação } & \multirow{2}{*}{$\begin{array}{l}\text { Coeficiente de } \\
\text { determinação }\end{array}$} & \multirow{2}{*}{ GL } & \multicolumn{2}{|c|}{ S.Q. Resíduo } \\
\hline & & & & Escala original & Equação estimada (Sf) \\
\hline \multicolumn{6}{|c|}{ Momordica charantia } \\
\hline $\mathrm{C}$ & Linear & 0,8754 & 98 & 4430,008 & $-0,1242+0,4699 \mathrm{C}$ \\
\hline $\mathrm{L}$ & Linear & 0,9085 & 98 & 3310,765 & $-0,1288+0,5971 \mathrm{~L}$ \\
\hline CL & Linear & 0,9460 & 98 & 1992,832 & $0,1053+0,4742 \mathrm{CL}$ \\
\hline $\operatorname{CL}(0,0)$ & Linear & 0,9460 & 99 & 2039,646 & $0,4963 \times \mathrm{CL}$ \\
\hline $\mathrm{C}$ & Geométrica & 0,8935 & 98 & 4169,652 & $0,7346 \times \mathrm{C}^{0,1675}$ \\
\hline $\mathrm{L}$ & Geométrica & 0,9143 & 98 & 3286,544 & $0,9335 \times \mathrm{L}^{0,1758}$ \\
\hline $\mathrm{C}$ & Exponencial & 0,9143 & 98 & 4879,928 & $0,2783 \times 0,1306^{\mathrm{C}}$ \\
\hline $\mathrm{L}$ & Exponencial & 0,8930 & 98 & 4744,670 & $0,2889 \times 0,1387^{\mathrm{C}}$ \\
\hline \multicolumn{6}{|c|}{ Pyrostegia venusta } \\
\hline $\mathrm{C}$ & Linear & 0,8912 & 98 & 1121,141 & $-0,7996+0,3289 \mathrm{C}$ \\
\hline $\mathrm{L}$ & Linear & 0,9337 & 98 & 698,856 & $-0,8659+0,7238 \mathrm{~L}$ \\
\hline $\mathrm{CL}$ & Linear & 0,9755 & 98 & 264,127 & $0,1278+0,6587 \mathrm{CL}$ \\
\hline CL $(0,0)$ & Linear & 0,9755 & 99 & 264,692 & $0,6649 \times \mathrm{CL}$ \\
\hline $\mathrm{C}$ & Geométrica & 0,9066 & 98 & 1031,229 & $0,4995 \times \mathrm{C}^{0,1731}$ \\
\hline $\mathrm{L}$ & Geométrica & 0,9417 & 98 & 533,1271 & $0,1777 \times \mathrm{L}^{0,1770}$ \\
\hline $\mathrm{C}$ & Exponencial & 0,9417 & 98 & 1635,268 & $0,1552 \times 0,1376^{\mathrm{C}}$ \\
\hline $\mathrm{L}$ & Exponencial & 0,9253 & 98 & 986,724 & $0,1487 \times 0,2003^{\mathrm{L}}$ \\
\hline
\end{tabular}

1/ em que C e L correspondem a comprimento e largura, respectivamente.

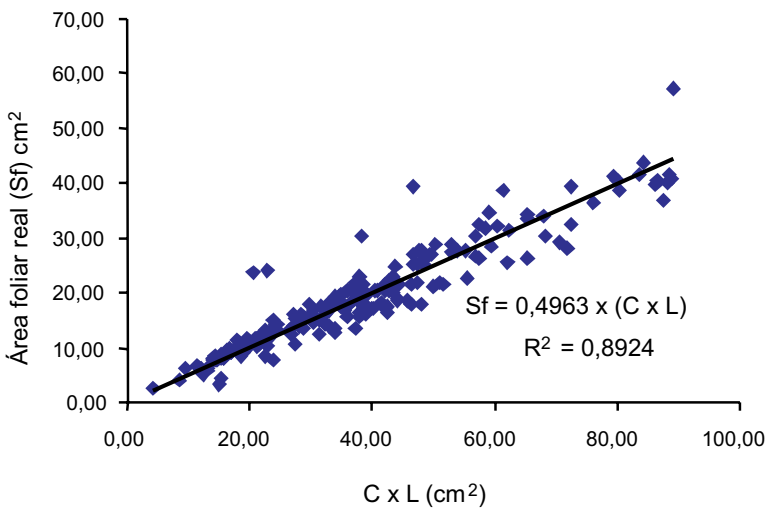

Figura 1 - Regressão linear simples entre a área foliar real e o produto do comprimento (C) pela largura (L) dos folíolos de Momordica charantia. FCAV/UNESP, Jaboticabal-SP, 2007.

relação às retas obtidas, sugerindo que as equações apresentadas podem representar a área foliar real de maneira satisfatória (Figuras 1 e 2). As equações obtidas neste trabalho são ferramentas importantes para o estudo da análise de crescimento dessas espécies, uma vez que o cálculo da área foliar é imprescindivel para a realização desses estudos.

Planta Daninha, Viçosa-MG, v. 27, n. 4, p. 683-687, 2009

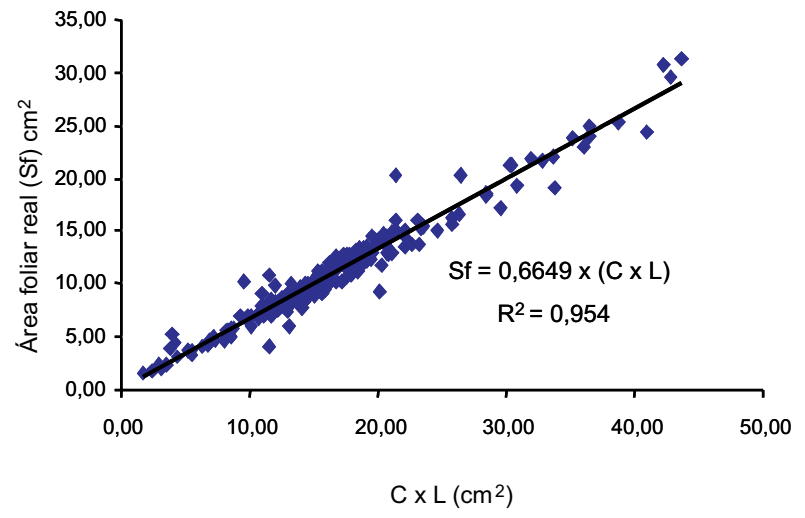

Figura 2 - Regressão linear simples entre a área foliar real e o produto do comprimento (C) pela largura (L) dos folíolos de Pyrostegia venusta. FCAV/UNESP, Jaboticabal-SP, 2007.

Os resultados do presente trabalho permitem concluir que todas as equações obtidas podem ser utilizadas para estimar a área foliar de Momordica charantia e Pyrostegia venusta. Contudo, utilizando as equações lineares passando pela origem, $\mathrm{S}_{\mathrm{f}}=0,4963 \times(\mathrm{C} \times \mathrm{L})$ para Momordica charantia e $\mathrm{S}_{\mathrm{f}}=0,6649 \times(\mathrm{C} \times \mathrm{L})$ para Pyrostegia venusta foram as que apresentaram menores erros nas estimativas. 


\section{LITERATURA CITADA}

BENINCASA, M. M. P. et al. Método não destrutivo para estimativa de área foliar de Phaseolus vulgaris L. (feijoeiro) Científica, v. 4, n. 1, p. 43-48, 1976

BIANCO, S. et al. Estimativa de área foliar de plantas daninhas. XIII - Amaranthus retroflexus L. Ecossistema, v. 20, n. 1, p. $5-9,1995$

BIANCO, S. et al. Estimativa da área foliar de Ipomoea hederifolia e Ipomoea nil Roth. usando dimensões lineares do limbo foliar. Planta Daninha, v. 25, n. 2, p. 325-329, 2007.

CARVALHO, S. J. P.; CHRISTOFFOLETI, P. J. Leaf area estimation of five Amaranthus species using leaf blade linear dimensions. Planta Daninha, v. 25, n. 2, p. 317-324, 2007.

DAVIS, J. F. The relationship between leaf area and yield of the field bean with a statistical study of methods for determining leaf area. J. Am. Soc. Agron., v. 32, n. 5, p. 323-329, 1940.

FERREIRA, D. T.; ALVARES, P. S. M.; HOUGHTON, P. J. Chemical constituents from roots of Pyrostegia venusta and considerations about its medicinal importance. Química Nova, v. 23, n. 1, p. 42-46, 2000.
KISSMAN, K. G.; GROTH, D. Plantas infestantes e nocivas. São Paulo: BASF Brasileira, 1999. Tomo II. p. $746-748$.

LORENZI, H. Plantas daninhas do Brasil: terrestres, aquáticas, parasitas e tóxicas. Nova Odessa: Instituto Plantarum, 2008. p. 192-261.

LUCCHESI, A. A. Utilização prática de análise de crescimento vegetal. An. ESALQ, v. 41, n. 1, p. 181-202, 1984.

MEAD, R.; CURNOW, R. N. Statistical methods in agriculture and experimental biology. New York: Chapman and Hall, 1983. p. 335.

NETER, J.; WASSERMAN, W. Applied models. Regressions, analysis of variance and experimental designs. Illinois: Rechard D. Irwin, 1974. p. 842.

PERESSIN, V. A.; PITELLI, R. A.; PERECIN, D. Métodos para estimativa da área foliar de plantas daninhas. 4. Cassia tora L. Planta Daninha, v. 7, n. 2, p. 48-52, 1984.

SILVA, N. F. et al. Modelos para estimar a área foliar de abóbora por meio de medidas lineares. R. Ceres, v. 45, n. 259 , p. 287-291, 1998. 